\title{
Quantized Transport in Graphene p-n Junctions in Magnetic Field
}

\author{
D. A. Abanin and L. S. Levitov \\ Department of Physics, Massachusetts Institute of Technology, 77 Massachusetts Ave, Cambridge, MA 02139
}

\begin{abstract}
Recent experimental work on locally gated graphene layers resulting in p-n junctions have revealed quantum Hall effect in their transport behavior. We explain the observed conductance quantization which is fractional in the bipolar regime and integer in the unipolar regime in terms of quantum Hall edge modes propagating along and across the p-n interface. In the bipolar regime the electron and hole modes can mix at the p-n boundary, leading to current partition and quantized shot noise plateaus similar to those of conductance, while in the unipolar regime transport is noiseless. These quantum Hall phenomena reflect the massless Dirac character of charge carriers in graphene, with particle-hole interplay manifest in mode mixing and noise in the bipolar regime.
\end{abstract}

The transport properties of graphene, 2-dimensional sheets of graphite [1], in particular the high carrier mobility and tunability of transport characteristics, make this material attractive for applications in nanoelectronics 2, [3]. Various methods have been developed for patterning graphene sheets into prototype devices such as quantum dot transistors [1] and nanoribbons [4, [5], followed by demonstration of local control of carrier density in a graphene sheet [6]. Besides possible device applications, graphene junctions are predicted to host new and exciting phenomena reflecting massless Dirac character of carriers in this material, such as Klein tunneling 7], particle collimation [8], quasibound states [9], and Veselago lensing [10]. In addition, interesting phenomena are expected in gated graphene bilayers, where the field-effect transport can be induced by tuning the gap at the Dirac point [11]. These applications make gating of graphene a topic of great interest.

Recently, a graphene p-n junction with individual control of carrier density in two adjacent regions with a pair of gates above and below it was reported [12]. The density in each region could be varied across the neutrality point, allowing pn, pp and nn junctions to be formed at the interface. The interface width was quite small owing to $30 \mathrm{~nm}$ distance to the top gate and its sharp edge. Transport measurements, carried out in the quantized Hall effect (QHE) regime at fields $3 \mathrm{~T}<B<8 \mathrm{~T}$, revealed ohmic two-terminal conductance taking quantized values $g=6,2,3 / 2,1$ in the units of $e^{2} / h$. Along with the QHE plateaus with $g=2$ and 6 in the unipolar regime, quantized plateaus $g=1$ and $3 / 2$ of similar quality were observed in the bipolar regime. While conductance of $6 \frac{e^{2}}{h}$ and $2 \frac{e^{2}}{h}$ is a hallmark of the integer QHE in graphene [13, 14], quantized conductance values $3 / 2$ and 1 are unusual and call for explanation.

We interpret these observations by linking them to the properties of the Dirac-like carriers, which give rise to bipolar, electron and hole, QHE edge modes at the pn interface (Fig 1). The behavior at the interface is explained by employing ideas from the theory of quantumchaotic transport [15, 16, 17, 18, 19, 20]. Although in our case the edge modes carry charge along the pn interface
A

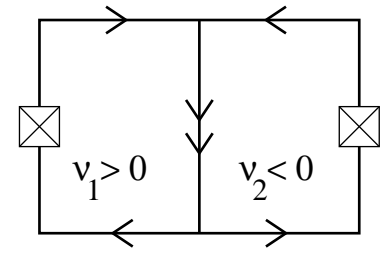

FIG. 1: Schematic of QHE edge states for (A) bipolar regime, and $(\mathbf{B})$ unipolar regime of graphene junction. In case $(\mathbf{A})$ the edge states counter-circulate in the $\mathrm{n}$ and $\mathrm{p}$ regions, bringing to the pn interface electrons and holes from different reservoirs. Mode mixing at the interface leads to the two-terminal conductance (1). In case (B), since the edge states circulate in the same direction without backscattering or mixing, conductance is determined by the modes permeating the whole system, $g=\min \left(\left|\nu_{1}\right|,\left|\nu_{2}\right|\right)$.

all in the same direction, in a chiral rather than chaotic fashion, we argue that inter-mode scattering within the pn interface region gives rise to dynamics with features analogous to those known for quantum-chaotic systems.

In this analogy the QHE states at the sample boundary play the role of perfect lead channels of chaotic quantum dots 15, 16], bringing charge to the pn interface and carrying it away into reservoirs. However, several physical effects causing conductance fluctuations in chaotic dots are absent in our case, leading to quantization of twoterminal conductance not known for the dots. In particular, the effective lead channels are quantized more perfectly than in the dots, owing to backscattering suppression in QHE transport. In addition, the quantummechanical interference effects which lead to samplespecific conductance fluctuations, can be suppressed in our case due to self-averaging, as well as dephasing and electron-electron scattering. Other effects that can affect the edge state transport at the pn interface are intermode relaxation and coupling to electronic states in QHE bulk, causing dephasing in a manner similar to the voltage probe model [21]. While these regimes yield similar results for conductance, they will manifest themselves differently in other characteristics, in particular in electron shot noise [22], which can be used for detailed char- 


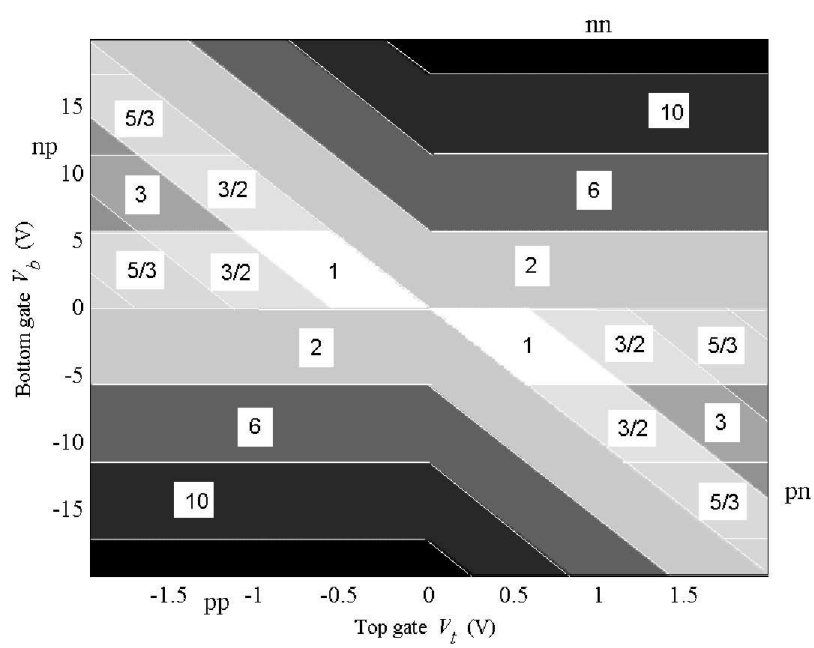

FIG. 2: Two-terminal conductance vs. gate voltage, given by Eq. 1 in the bipolar case $\nu_{1}>0, \nu_{2}<0$, and by Eq. 2 in the unipolar case $\left(\nu_{1,2}\right.$ of equal sign). The boundaries of QHE regions are specified by $\nu_{1,2}=0, \pm 4, \pm 8 \ldots$, with the gate voltage dependence of $\nu_{1,2}$ given by Eq.3. Parameters used: distances to the top and back gates $h=30 \mathrm{~nm}, d=300 \mathrm{~nm}$, magnetic length $\ell_{B}=10 \mathrm{~nm}$, dielectric constant $\kappa=3$.

acterization of transport mechanisms.

Due to particle-hole symmetry of carriers in graphene, the QHE in this material occurs symmetrically about the neutrality point at the densities $\nu= \pm 2, \pm 6, \pm 10 \ldots[13$, 14]. In each of these quantized states there are $n=|\nu|$ edge modes propagating in different directions at $\nu>0$ and $\nu<0[23,24]$. For the bipolar case, assuming QHE at densities $\nu_{1}>0$ and $\nu_{2}<0$ on either side of the boundary, this gives $\left|\nu_{1}\right|$ and $\left|\nu_{2}\right|$ edge modes circulating in opposite directions that merge to form a multi-mode edge state at the pn interface (Fig,1A). These modes supply to the pn interface particles from both the $\mathrm{n}$ and $\mathrm{p}$ reservoirs. After propagating together along the interface these particles arrive at the sample boundary where they are ejected into the edge modes which split up and return to reservoirs.

The observed conductance quantization can be readily explained by assuming full mixing of these modes at the pn interface, such that for each particle the probability to be ejected into any of the $N=\left|\nu_{1}\right|+\left|\nu_{2}\right|$ modes equals $p_{N}=1 / N$ irrespective of its origin. The two-terminal conductance is then obtained by multiplying $p_{N}$ by the numbers of the modes, giving

$$
g_{\mathrm{pn}}=\frac{\left|\nu_{1}\right|\left|\nu_{2}\right|}{\left|\nu_{1}\right|+\left|\nu_{2}\right|}=1, \frac{3}{2}, 3, \frac{5}{3} \ldots
$$

where $\nu_{1,2}= \pm 2, \pm 6, \pm 10 \ldots$. This agrees with the observed quantized values [12] (see Fig 22).

The character of QHE edge transport in the unipolar regime is quite different. In this case, nn or pp, the

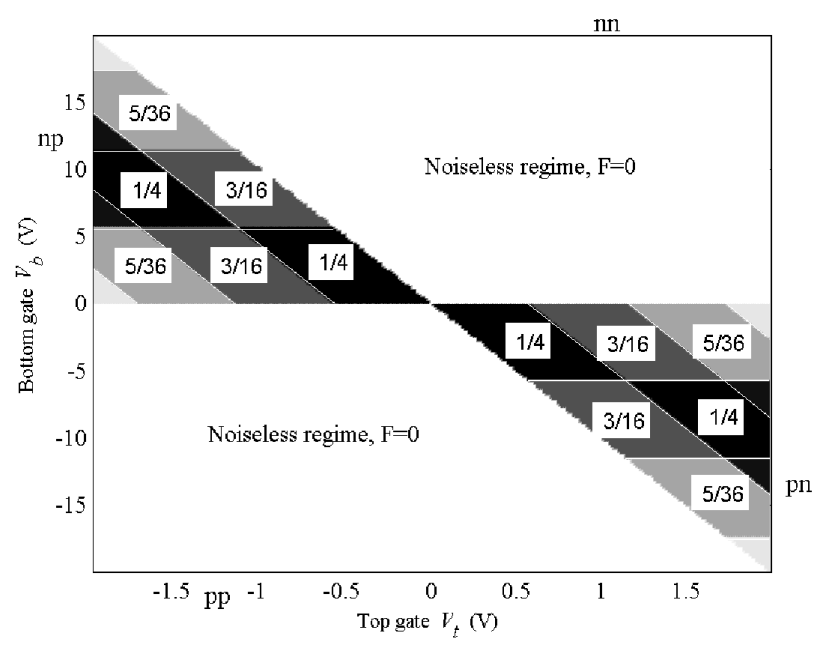

FIG. 3: Shot noise Fano factor, Eq.8, plotted vs. gate voltages for the same parameter values as in Fig 2, Noise is zero in the unipolar regime ( $\mathrm{pp}$ or $\mathrm{nn}$ ) due the absence of current partition at the junction interface, but finite in the bipolar regime due to edge mode mixing at the pn interface.

edge modes in both regions circulate in the same direction. As a result, some modes are coupled to both reservoirs, while the others are connected to only one of the reservoirs (Fig 1B B). With backscattering suppressed by QHE, the conductance across the boundary is solely due to those edge modes that permeate the entire system, making contact with both reservoirs. This gives the observed nonclassical conductance values

$$
g_{\mathrm{nn}}=g_{\mathrm{pp}}=\min \left(\left|\nu_{1}\right|,\left|\nu_{2}\right|\right)=2,6,10 \ldots
$$

$\nu_{1,2}= \pm 2, \pm 6, \pm 10 \ldots$, in agreement with the known results for quantized conductance of constrictions between different QHE states 25, 26]. The nondissipative character of transport in the unipolar regime, Eq.2, resulting from suppressed backscattering, can be revealed by measuring noise. In the absence of current partitioning inside the sample we expect only thermal Johnson-Nyquist noise $S=2 g k_{\mathrm{B}} T$ in this regime but no shot noise contribution (see Fig 3 ).

The conductance values given by Eqns.2 and 11 occur in a particular pattern [12] that can be described as follows (see Fig 21). Electron density in graphene induced by the back gate is $n_{1}=(\kappa / 4 \pi e) V_{\mathrm{b}} / d$, where $d$ is the distance to the gate, $V_{\mathrm{b}}$ is voltage on it, and $\kappa$ is dielectric constant. Similarly, in the locally gated region we have $n_{2}=(\kappa / 4 \pi e)\left(V_{\mathrm{b}} / d+V_{\mathrm{t}} / h\right)$, where $h$ and $V_{\mathrm{t}}$ are the distance to the top gate and voltage on it. For the Landau level filling factors $\nu_{1,2}=(h c / e B) n_{1,2}$ we find

$$
\nu_{1}=\left(\ell_{B}^{2} \kappa / 2 e\right) V_{\mathrm{b}} / d, \quad \nu_{2}=\left(\ell_{B}^{2} \kappa / 2 e\right)\left(V_{\mathrm{b}} / d+V_{\mathrm{t}} / h\right),
$$

with $\ell_{B}=\sqrt{\hbar c / e B}$ the magnetic length. The values $V_{\mathrm{b}}$, 
$V_{\mathrm{t}}$ corresponding to integer QHE states are inside parallelograms with the boundaries approximately given by $\nu_{1,2}=0, \pm 4, \pm 8 \ldots$, as appropriate for the four-fold degenerate graphene Landau levels 13, 14]. The resulting conductance pattern, shown in Fig 2 for realistic parameter values, strikingly resembles the experimental results [12].

How is the conductance in Eq.11 affected by quantummechanical interference effects? Random matrix theory (RMT) of chaotic transport predicts [15, 16] ensembleaveraged conductance $\bar{g}=n_{1} n_{2} /\left(n_{1}+n_{2}+1-2 / \beta\right)$, where $n_{1,2}$ is the open channel number, and $\beta=1,2,4$ for the three random matrix universality classes. In our QHE case, with the channel numbers $n_{1,2}=\left|\nu_{1,2}\right|$ and $\beta=2$, RMT predicts $\bar{g}$ identical to Eq.1. Similarly, semiclassical description of transport in chaotic cavities 17], where mixing is due to the dynamics in the cavity, yields conductance values close to the classical result for two conductors connected in series.

To clarify the origin of the mode mixing at the pn interface we studied electron density distribution for the gate geometry used in Ref. [12]. Numerical solution of Laplace problem for the electrostatic potential in between the gates revealed that the pn density step is about $40 \mathrm{~nm}$ wide, a few times larger than the magnetic length at $B=8 \mathrm{~T}$. Comparison to the known results [27] for a compressible region sandwiched between incompressible regions then suggests the presence at the pn interface of additional QHE modes that, in the presence of disorder, can facilitate inter-channel scattering and mixing.

In the fully coherent regime conductance would exhibit universal fluctuations, UCF. The magnitude of UCF predicted for chaotic transport (see Ref. [20]) in our case depends on the channel numbers as follows:

$$
\operatorname{var}(g)=\frac{\nu_{1}^{2} \nu_{2}^{2}}{\left(\left|\nu_{1}\right|+\left|\nu_{2}\right|\right)^{2}\left(\left(\left|\nu_{1}\right|+\left|\nu_{2}\right|\right)^{2}-1\right)} .
$$

Applied to the observed plateaus with $\left(\nu_{1}, \nu_{2}\right)=$ $(2,-2),(2,-6),(6,-2)$, Eq.4 indicates that these plateaus would not have been discernible in a system with fully developed UCF. We therefore conclude that the observed quantization of $g$ depends on some mechanism that suppresses UCF. For example, the suppression could easily be understood if Thouless energy for the states at the pn interface was small compared to $k_{\mathrm{B}} T$. The reduced $\mathrm{UCF}$ would then result from averaging over the $k_{\mathrm{B}} T$ energy interval. However, the plateaus in 12 . remain unchanged when temperature is reduced from $4 \mathrm{~K}$ to $250 \mathrm{mK}$, making such a scenario unlikely.

The UCF suppression may signal a fundamental departure of chiral QHE dynamics from that of the earlier studied systems. However, at this point we cannot exclude other, more mundane explanations. In particular, time-dependent fluctuations of system parameters can supercede mesoscopic fluctuations, turning the observed time-averaged quantities into ensemble-averaged quantities. This self-averaging could arise naturally due to fluctuating electric field at the pn interface induced by voltage noise on the gates. Another, more interesting explanation could be that UCF suppression indicates presence of dephasing due to the coupling of the chiral modes to the localized states in the bulk, or some other intrinsic mechanism.

Current partition due to mode mixing at the pn interface will manifest itself in the finite shot noise intensity. To evaluate noise, we note that mixing of the reservoir distributions, no matter of what origin, results in particle energy distribution of the form

$$
n(\varepsilon)=\frac{\left|\nu_{1}\right|}{N} n_{1}(\varepsilon)+\frac{\left|\nu_{2}\right|}{N} n_{2}(\varepsilon)
$$

which at small $k_{\mathrm{B}} T$ is a double step. In analogy with diffusive systems [28], and chaotic cavities [17, 19], this distribution serves as a Kogan-Shulman-like extraneous source of current fluctuations,

$$
J=\int n(\varepsilon)(1-n(\varepsilon)) d \varepsilon=\frac{\left|\nu_{1}\right|\left|\nu_{2}\right|}{N^{2}}\left|V_{s d}\right| .
$$

We relate the noise source $J$ to the fluctuations of the two-terminal current by noting that, since fluctuating current of intensity $J$ is injected into each open channel, the current fluctuations flowing into the $\mathrm{n}$ and $\mathrm{p}$ regions will be $J_{1}=\left|\nu_{1}\right| J$ and $J_{2}=\left|\nu_{2}\right| J$. Converting these fluctuations into voltage fluctuations and adding the contributions of the $\mathrm{n}$ and $\mathrm{p}$ regions, we find the voltage fluctuations induced between the reservoirs:

$$
\left\langle\delta V^{2}\right\rangle=\frac{J_{1}}{\left|\nu_{1}\right|^{2}}+\frac{J_{2}}{\left|\nu_{2}\right|^{2}}=\left(\frac{1}{\left|\nu_{1}\right|}+\frac{1}{\left|\nu_{2}\right|}\right) J=\frac{\left|V_{s d}\right|}{N} .
$$

Current noise can now be obtained as $S=g^{2} \delta V^{2}$, where $g$ is the conductance (11). It is convenient to characterize noise by the Fano factor $F=S / I$, describing noise suppression relative to Poisson noise. We find

$$
F=\frac{\left|\nu_{1}\right|\left|\nu_{2}\right|}{\left(\left|\nu_{1}\right|+\left|\nu_{2}\right|\right)^{2}}=\frac{1}{4}, \frac{3}{16}, \frac{5}{36} \ldots
$$

where $\nu_{1,2}=2,6,10 \ldots$. The result (8) is identical in form to the shot noise Fano factors of chaotic cavities [17, 19]. The Fano factor values (8) should be contrasted with $F \approx 0.29$ predicted for a $\mathrm{p}-\mathrm{n}$ junction in the absence of magnetic field [8].

Another regime for noise is possible if electrons, while traveling along the pn interface, have enough time to transfer energy to each other via inelastic processes. This will occur if $\tau_{e l} \ll L / v$, where $\tau_{e l}$ is the characteristic electron energy relaxation time, $v$ is drift velocity and $L$ is the pn interface length. (A similar regime was analyzed for diffusive 28] and chaotic 19] transport.) In this case, the electron energy distribution is characterized by an effective temperature $T_{\text {eff }}$ which is determined by the 
balance of the energy supplied from reservoirs and electron thermal energy flowing out:

$$
\frac{1}{2} \frac{\left|\nu_{1}\right|\left|\nu_{2}\right|}{\left|\nu_{1}\right|+\left|\nu_{2}\right|} V_{s d}^{2}=\zeta(2)\left(\left|\nu_{1}\right|+\left|\nu_{2}\right|\right) k_{\mathrm{B}}^{2} T_{\mathrm{eff}}^{2},
$$

$\zeta(2)=\pi^{2} / 6$. The extraneous fluctuations, Eq, 6 , evaluated for the Fermi distribution with $T=T_{\text {eff }}$, give $J=k_{\mathrm{B}} T_{\text {eff }}$. Repeating the reasoning that has led to Eq. 8 we find the noise intensity $S=g k_{\mathrm{B}} T_{\text {eff }}$. This expression resembles the Nyquist formula, except for the factor of two missing because the fluctuations (6) occur only in the pn region but not in the leads. Since $T_{\text {eff }} \propto V_{s d}$, this noise is linear in $V_{s d}$. Similar to the $T=0$ shot noise, we characterize it by Fano factor $\tilde{F}=(3 F)^{1 / 2} / \pi$, with $F$ given by Eq, 8

We finally note that noise can be used to test which of the UCF suppression mechanisms discussed above, selfaveraging or dephasing, occur in experiment [12]. For coherent transport noise exhibits mesoscopic fluctuations similar to UCF which can be analyzed within RMT framework. In the absence of time reversal symmetry, RMT yields ensemble-averaged Fano factor

$$
\bar{F}=\frac{\left|\nu_{1}\right|\left|\nu_{2}\right|}{\left(\left|\nu_{1}\right|+\left|\nu_{2}\right|+1\right)\left(\left|\nu_{1}\right|+\left|\nu_{2}\right|-1\right)}
$$

(see Eq. 11 in Ref. [20]). For $\nu_{1,2}=2,4,6 \ldots$ this gives $\bar{F}=4 / 15,12 / 63,36 / 143 \ldots$. These values, expected when transport is coherent but self-averaged, are different from Eq 8 obtained from incoherent mixing model.

The quantized transport observed in graphene pn junctions [12] is of different character in the unipolar and bipolar regimes. In the first case, transport is dissipationless with conductance quantized to an integer. In the second case, mode mixing at the pn interface creates a situation similar to that studied in the quantumchaotic transport. Conductance quantized to fractional values observed in Ref. [12] then results from intrinsic or extrinsic suppression of UCF. These transport regimes can be unraveled using electron shot noise, predicted to be finite in the bipolar regime and zero in the unipolar regime, with quantized plateau structure similar to that of conductance.

We benefited from discussions with C. M. Marcus, L. Di Carlo, J. R. Williams, P. Kim and P. Jarillo-Herrero, and from comments made by Y. M. Blanter and E. V. Sukhorukov. This work is supported by NSF MRSEC (DMR 02132802) and NSF-NIRT DMR-0304019.
[1] A. K. Geim, K. S. Novoselov, Nat. Mater. 6, 183 (2007).

[2] Y.-W. Son, M. L. Cohen, S. G. Louie, Nature 444, 347 (2006).

[3] A. Rycerz, K. J. Tworzydlo, C. W. J. Beenakker, Nat. Phys. 3, 172 (2007).

[4] Z. Chen, Y.-M. Lin, M. J. Rooks, P. Avouris, available at http://arXiv.org/abs/cond-mat/0701599

[5] M. Y. Han, B. Oezyilmaz, Y. Zhang, P. Kim, available at http://arXiv.org/abs/cond-mat/0702511

[6] B. Huard et al., available at http://arXiv.org/abs/0704.2626.

[7] M. I. Katsnelson, K. S. Novoselov, A. K. Geim, Nat. Phys. 2, 620 (2006).

[8] V. V. Cheianov, V. I. Falko, Phys. Rev. B 74, 041403 (2006).

[9] P. G. Silvestrov, K. B. Efetov, Phys. Rev. Lett. 98, 016802 (2007).

[10] V. V. Cheianov, V. I. Fal'ko, B. L. Altshuler, Science 315, 1252 (2007).

[11] T. Ohta, A. Bostwick, T. Seyller, K. Horn, E. Rotenberg, Science 313, 951 (2006).

[12] J. Williams, L. Di Carlo, C. M. Marcus, available at http://arXiv.org/abs/0704.3487.

[13] K. S. Novoselov et al., Nature 438, 197 (2005).

[14] Y. Zhang, Y.-W. Tan, H. L. Stormer, P. Kim, Nature 438, 201 (2005).

[15] C. W. J. Beenakker, Rev. Mod. Phys. 69, 731 (1997).

[16] H. U. Baranger, P. A. Mello, Phys. Rev. Lett. 73, 142 (1994).

[17] Ya. M. Blanter, E. V. Sukhorukov, Phys. Rev. Lett. 84, 1280 (2000).

[18] O. Agam, I. Aleiner, A. I. Larkin, Phys. Rev. Lett. 85, 3153 (2000).

[19] S. Oberholzer et al., Phys. Rev. Lett. 86, 2114 (2001).

[20] D. V. Savin, H.-J. Sommers, Phys. Rev. B 73, 081307(R) (2006).

[21] M. Büttiker, Phys. Rev. B 38, 9375 (1988).

[22] Ya. M. Blanter, M. Büttiker, Phys. Rep. 336, 1 (2000).

[23] N. M. R. Peres, F. Guinea, A. H. Castro Neto, Phys. Rev. $B$ 73, 125411 (2006).

[24] D. A. Abanin, P. A. Lee, L. S. Levitov, Phys. Rev. Lett. 96, 176803 (2006).

[25] R. J. Haug, A. H. MacDonald, P. Streda, K. von Klitzing Phys. Rev. Lett. 61, 2797 (1988).

[26] S. Washburn, A. B. Fowler, H. Schmid, D. Kern, Phys. Rev. Lett. 61, 2801 (1988).

[27] A. V. Khaetskii, V. I. Fal'ko, G. E. W. Bauer, Phys. Rev. $B$ 50, 4571 (1994).

[28] K. E. Nagaev, Phys. Lett. A 169, 103 (1992). 